\title{
A Systems Science Perspective on Addressing Obesity in the Veterans Health Administration
}

\author{
Nicolaas P. Pronk, PhD, MA ${ }^{1,2}$ \\ ${ }^{1}$ HealthPartners Institute, Bloomington, MN, USA; ${ }^{2}$ Department of Social and Behavioral Sciences, Harvard T.H. Chan School of Public Health, \\ Boston, MA, USA.
}

J Gen Intern Med 32(Suppl 1):S9-S10

DOI: $10.1007 / \mathrm{s} 11606-016-3975-9$

(c) Society of General Internal Medicine 2017

$\mathrm{T}$ he recognition of the full spatial and temporal context in which our genes are placed at once both appreciates and acknowledges the complexity of life. Our genes have an innate capacity to respond to environmental stimuli; a wondrous plasticity and dynamic evolving developmental process that facilitates change over time. Recognition of this concept also points to the notion that both environment and genetics matter-it's a both-and, not an either-or. From a systems science perspective, the environment in which our genes find themselves is affected by a multitude of stimuli that stem from the intra-individual biological and psychological situations, the behaviors in which people engage, the external multilevel milieu people are exposed to, and the multitude of interactions and interdependencies of all of those factors. This dynamic, adaptive, non-linear response to our environment shapes who we are at a given moment in time. Activity that reflects everything we do, from genetic plasticity to responses to environmental stimuli, makes us - in essence-become what we do.

\section{OBESITY AND SYSTEMS SCIENCE}

Obesity at the individual and population levels may be considered the net result of the complex multifactorial interplay among a set of varied and diverse factors such as past experiences, individual behaviors, family dynamics, workplace culture, community design, environmental exposures, and state and federal policy. ${ }^{1}$ Systems science represents a broad approach that recognizes the importance of the whole and the interdependence of its component parts. Various methodologies may be applied to study the dynamic properties of system components, which may involve multiple scales (e.g., levels spanning cells [micro], individuals and families [meso], to society [macro]), while simultaneously studying the behavior of the system as a whole. ${ }^{1-4}$ Increasingly, approaches for addressing obesity call for systems science perspectives to inform decision-

Published online March 7, 2017 making regarding resource investments and policy development. ${ }^{1,3,4}$

Obesity has proven itself to be an "unrelenting challenge," highly resistant to interventions. As a result, the prevalence of obesity has steadily increased, and it now affects more than one third of the US population. ${ }^{5}$ More specific to the Veterans Health Administration (VHA), more than $40 \%$ of patients receiving care within the VHA are obese. ${ }^{6}$ However, the burden among those treated at the VHA appears to be disproportionately related to excess weight, as $77 \%$ of veterans who present as patients to the health system are overweight or obese, compared to $68 \%$ of the general population. ${ }^{6}$

\section{OBESITY INTERVENTIONS AT THE VHA}

The recent State of the Art Conference (SOTA) on Weight Management in the VHA shows the tremendous challenge obesity presents to both patients and the VHA system, and that this challenge requires an integrated and coordinated approach. ${ }^{7}$ Various well-designed and quality options are available to patients with obesity who present at the VHA, including weight loss surgery, pharmacotherapy, and behavioral treatment. Each of these treatment options has shown effectiveness in generating weight loss among those who participate; however, participation in these solutions among the approximately 4 million overweight or obese veterans who receive VHA care remains a significant challenge. In 2015, more than 135,000 veterans had at least a single interaction with the behavioral MOVE! Weight Management Program, approximately 315 veterans underwent bariatric surgery procedures, and approximately 2700 veterans were prescribed a weight loss medication annually from 2012 to 2015 . Hence, a reasonable estimate of penetration into the patient population may be about 3 to $5 \%$.

The recent SOTA on Weight Management in the VHA has generated several conclusions, including the recommendations for the VHA to establish a vision that supports healthy weight for all veterans, to identify a metric for assessing impact across the VHA system, and to learn how to reliably implement and scale effective solutions for preventing and treating obesity. ${ }^{7}$ These recommendations lend themselves well to a systems science approach. 


\section{A Vision for Health and Well-Being}

Obesity is merely one aspect of a person's overall health and well-being. Health, in fact, is not an end in itself. Rather, it represents a means to what people actually want to do or achieve in life. Hence, health may be considered part of overall, subjective well-being. In this context, obesity may be positioned as part of an overall effort to promote overall health and well-being. ${ }^{8}$ Such a vision may support the VHA in creating a framework that allows for the integration of comprehensive programming with a focus on what really matters to people. Obesity treatment in this context may take on a very different approach from one with a singular focus on energy balance or medical treatment. It may shape itself around the need for social connections, educational pursuits, a change in physical environment, or a shift towards healthy lifestyles, just to name a few. From a VHA health system perspective, several systems characteristics will propagate and support such a vision. These characteristics include leadership, values, and connections.

- Leadership and values - across all levels of the organization, leadership needs to live the values and provide permission for all stakeholders to engage in their work to support patients. In the absence of clear values, support functions (e.g., measurement or finance) can sometimes fill the void and become de facto values. Unfortunately, such practices would actually increase system stress. Rather, emphasis should be placed on de-stressing values such as transparency, compassion, partnership, or integrity. ${ }^{9}$

- Connections - Increasing the connections and networks that provide positive support to all stakeholders may have a profound impact on both provision and receipt of obesity prevention and management solutions. Such networks and their density reflect integrated capacity for partnerships.

\section{A Metric to Monitor System's Progress}

A simple metric organized around the need to measure impact has the potential to generate alignment of effort across an entire system. Optimally, such a metric should reflect a value proposition - a ratio of benefits over costs. When considering obesity, such a metric could be the result of computational and simulation modeling that reflects the complexity of obesity and the design of the available interventions and policies to address it. Systems-level metrics that allow change efforts to be monitored will ensure continued focus and will allow for meaningful process improvements to occur.

\section{A Scalable and Sustainable Process}

Randomized controlled trials may show successful outcomes, but translating such trials into real-world programmatic solu- tions may be extremely difficult. Especially for large populations, scalable and sustainable solutions are needed. ${ }^{10}$ Furthermore, scaled efforts must not lose their effectiveness. Sustainability of solutions demands a business plan that shows value and refers directly back to proposed metric described above.

\section{CONCLUDING THOUGHTS}

Addressing obesity within the VHA system is complex. Not recognizing or appreciating such complexity will drive suboptimal solutions. Systems science provides an opportunity to build integrated and comprehensive support systems for both patients and providers within the VHA to address obesity and overall health and well-being. Some components of an integrated support system will need to address the effectiveness and scalability of weight-related interventions. Other components will need to consider the vision needed to effectively address obesity and how to transform the workplace culture to achieve optimal results.

Acknowledgements: No external funding was received in the preparation of this paper, and the author reports no conflicts of interest.

Corresponding Author: Nicolaas P. Pronk, PhD, MA; HealthPartners Institute, Bloomington, MN, USA (e-mail: nico.p.pronk@healthpartners. com).

\section{REFERENCES}

1. Pronk NP, Narayan KMV. The application of systems science to addressing obesity at the workplace. J Occup Environ Med. 2016;58(2):123-126.

2. Bar-Yam Y. Making Things Work: Solving Complex Problems in a Complex World. NECSI, Cambridge, MA: Knowledge Press; 2004.

3. IOM (Institute of Medicine). Bridging the evidence gap in obesity prevention: a framework to inform decision making. Washington, DC: National Academies Press; 2010.

4. IOM (Institute of Medicine). Evaluating Obesity Prevention Efforts: A Plan for Measuring Progress. Washington, DC: The National Academies Press; 2013.

5. Ogden CL, Carroll MD, Kit BK, Flegal KM. Prevalence of childhood and adult obesity in the United states, 2011-2012. JAMA. 2014;311:806-814.

6. VA/DoD 2014 Clinical Practice Guideline for the Screening and Management of Overweight and Obesity. Available at: http://www.healthquality. va.gov/guidelines/cd/obesity/. Accessed 7 Dec 2016.

7. Raffa SD, Maciejewski MM, Zimmerman LE, et al. A system-level approach to overweight and obesity in the Veterans Health Administration. J Gen Intern Med. 10.1007/s11606-016-3948-Z

8. Kottke TE, Stiefel M, Pronk NP. "Well-being in all policies": promoting cross-sectoral collaboration to improve people's lives. Prev Chronic Dis. 2016;13:160155. doi:10.5888/pcd13.160155.

9. Ankel F, Noltimier M. Resilient \#MedEd \#Leaders: Identifying and Communicating Clear Values (Part 1). Available at: https://icenetblog. royalcollege.ca/2016/06/07/resilient-meded-leaders-identifying-andcommunicating-clear-values-part-1/ Accessed 5 Dec 2016.

10. Aziz Z, Absetz P, Oldroyd J, Pronk NP, Oldenburg B. A systematic review of real-world diabetes prevention programs: learnings from the last 15 years. Implement Sci. 2015;10:172. doi:10.1186/s13012-0354-6. 